\title{
Application of Fuzzy Neural Networks on Missile Launcher Fault Prediction
}

\author{
Cai Guifang \\ The Department of Aeronautics Ammunition Engineer \\ The First Aeronautics College of Air Force \\ Xinyang, China \\ e-mail: 18937669788@163.com
}

\begin{abstract}
In order to improve the reliability, security and validity of such type Missile Launcher, The faults prediction theory is introduced based on fuzzy neural network. The faults prediction analysis model is built, and an advanced training algorithm is proposed. Such algorithm is tested by using in the fault prediction of the missile launcher. The Experiment results illustrate that by using the proposed model, the faults prediction speed is faster $\mathbf{8 0} \%$ than ever, the prediction veracity is advanced, and the cost is reduced to $50 \%$.
\end{abstract}

Keywords-fuzzy neural network; fault prediction; arithmetic; fuzzy control; missile launcher.

\section{INTRODUCTION}

Nowadays Missile Launcher is one of the main battle weapon of such type battle plane, the faults of any part of it may reduce the system capability and even bring on bad accidents. So it is very important for guarantee the battle capability of the battle plane to guarantee the reliability, security and validity of the Missile Launcher effectively. Recently, by using and generalizing the faults diagnosis and prediction, the use and maintenance cost of many complicated devices is reduced obviously, the maintenance reliability and security is improved greatly. The application of faults diagnosis and prediction becomes more and more important. Because of the faults forepart detecting ability and high real timely diagnosis ability, the faults prediction technology has become one of the hot research directions [1]

The fuzzy neural network (FNN) is a kind of new technology which has the advantages of fuzzy theory and neural network technology. It can realize the fuzzy system's function by using the neural network's approach ability. At the same time, it has many capabilities such as the neural network' s low levels study, compute ability and fuzzy system' s high level study, decision-making ability [2-3]. So based on the fuzzy neural network, can predict the devices future states exactly.

\section{FAults PREDiction TheORY BASED ON FNN}

A neural network model built to train the natural dynamic course of a neural network expression system is called natural model, the structure of which is shown in Fig. 1 (a). The neural network is parallel connected with the distinguishing system, the outputs of which are the network's training signals. Such is a typical learning matter with

\author{
Hu Fanjun \\ The Department of Aeronautics Ammunition Engineer \\ The First Aeronautics College of Air Force \\ Xinyang, China \\ e-mail: 18937669169@163.com
}

teachers. The fact system as a teacher provides the needed anticipant outputs to the neural network arithmetic.

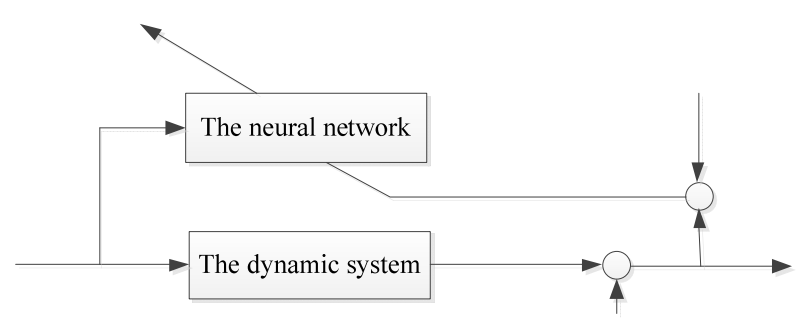

(a) Natural model structure

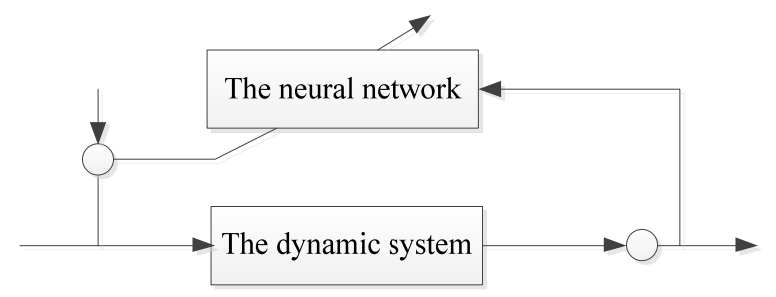

(b) Athwart model structure

Figure 1. Faults prediction modeling based on FNN

When the system is the controlled object or the traditional controller, the multilayer feedback neural network is adopted and the BP network or diversified transfigurations can be used directly. If the system is capability appraiser, the bestir arithmetic can be chose. Here multilayer perceptive component with all-around approach ability or cerebel model controller with part approach ability is alternately selected [4-5]. (b).

The dynamic system's athwart model is shown in Fig. 1

The athwart prediction's system outputs are the network inputs, comparing the network output with the system input, the corresponding input errors are used to be trained. So the system athwart model can be built based on the network learning. But if the non-linear system is irreversible, through the above method, a false athwart model will be found. So when building the system athwart models, the reversibility of the system should be guaranteed.

In order to obtain good athwart kinetic characteristics, the samples needed by net training should be chose carefully, it should have better run range than the actual run range of the 
sealed system. But in the real work, it is difficult to ascertain the input signals aforehand, and it is impossible to ascertain the sealed system's expectation output because the controlled object can make the system export expect movement. At the same time, in the prediction system, the definite durative invigorative input signals are used to ensure the coincident astringency of the parameter estimate arithmetic.

\section{FNN PREDICTION MODEL}

The if-then rules are adopted during the design course of the FNN prediction model [6]:

$R$ : If $x_{1}$ is $A_{\mathrm{p} 1}$ and $\ldots x_{\mathrm{n}}$ is $A_{\mathrm{pn}}$

Then $y_{1}$ is $B_{\mathrm{p} 1}$ and $y_{\mathrm{k}}$ is $B_{\mathrm{pk}}, \mathrm{p}=1,2, \ldots \mathrm{m}$

Among them $\mathrm{R}$ is the rule's symbol, $\mathrm{P}$ is the rules quantity, $\boldsymbol{x}=\left(\boldsymbol{x}_{1}, \boldsymbol{x}_{2}, \cdots \boldsymbol{x}_{n}\right)$ which is n-dimensions input vector, $B_{\mathrm{pk}}$ is a fuzzy aggregate of the output vectors.

Such FNN is a five-layer feedback neural network, the conterminous weight value; output and input are the fuzzy numbers.

The first layer is the input variable layer, which transmits the input value to the next layer:

$$
x_{i}^{(l)}=y_{i}^{(l)}=x_{i}^{(2)}
$$

In (1), $i$ is the quantity of the input variable, $i=1,2$, 3 ... The link value of the nerve cell is $w_{i}^{(l)}=1$.

The second layer is the fuzzing layer, the deferent dates are corresponding to the appurtenant of the input fuzzy muster. The common appurtenant functions are the trigonometric function, the trapezia function and the gauss function. The gauss function is adopted as the subject function:

$$
\begin{aligned}
& u_{i j}^{(2)}=\exp \left[\left(\frac{x_{i}-c_{i j}}{\sigma_{i j}}\right)^{2}\right] \\
& y_{i j}^{(2)}=\exp \left[\left(\frac{x_{i}-c_{i j}}{\sigma_{i j}}\right)^{2}\right]
\end{aligned}
$$

In (2) and (3), $u_{\mathrm{ij}}$ is the appurtenant degree function; $x_{i}$ is the input variable; $c_{\mathrm{ij}}$ is the center of the appurtenant function; $\sigma_{\mathrm{ij}}$ is the breadth of the appurtenant function; $1 \leqslant i \leqslant n$; $1 \leqslant j \leqslant m$; The link value of the nerve cell is $w_{i}^{(2)}=1$.

The third layer is the rule node, it represents the fuzzy rule, and the fuzzy rules base is made up all the fuzzy nodes of the third layer. This layer network can complete the matching of the fuzzy logic rule premise conditions, each node denotes to make the output of the second layer correlative each node carry fuzzy "AND" operation. The output is $y_{j}^{(3)}$.

$$
y_{j}^{(3)}=\sum_{i=1}^{n} x_{i j}^{(3)}=x_{i j}{ }^{(4)} \text {. }
$$

The link value of the nerve cell is $w_{i}^{(3)}=1$.

The formative dates of the fourth layer are corresponding to the output appurtenant functions, the output is $y_{j}^{(4)}$.

$$
y_{j}^{(4)}=w_{j}^{(4)}\left(p_{j} x_{1}+q_{j} x_{2}+r_{j} x_{3}+s_{j}\right)
$$

In (5), the link value of the nerve cell is $w_{i}^{(4)}$.

There is only one node in the fifth layer, the summation of all the input signals is calculated, then the system's output $\lg R$ is exported.

$$
y_{j}^{(5)}=\lg R=\frac{\sum_{i=1}^{n} w_{i} x_{i}}{\sum_{i=1}^{n} x_{i}}
$$

\section{FNN PREDICTION ATHMETIC}

\section{A. Prediction Athemetic}

The fuzzy analysis technology and FNN are adopted in the prediction method. The main athematic notion is: firstly grading the current diagnosis results of the expert system, secondly according to the graded results, carrying index trend prediction with the states of the future dates, at last according to the prediction results, carrying the next faults prediction with the fuzzy rules of the expert system. The arithmetic is as follow.

The error function is $E(k)$.

$$
E(k)=\frac{1}{2}(y-D)^{2}
$$

In (7), $y$ is the actually output, $D$ is the expect output, $k$ is the date sample serial number.

According to the grads descendant arithmetic, during the study process, the adjustment rules of $\omega_{i}, C_{i j}, \sigma_{i j}$ are as follow.

$$
\begin{aligned}
& \omega_{i}(k+1)=\omega_{i}(k)-\frac{\eta \partial E(k)}{\partial \omega_{i}(k)} \\
& C_{i j}(k+1)=C_{i j}(k)-\frac{\eta \partial E(k)}{\partial C_{i j}(k)}
\end{aligned}
$$




$$
\sigma_{i j}(k+1)=\sigma_{i j}(k)-\frac{\eta \partial E(k)}{\partial \sigma_{i j}(k)}
$$

In (8), (9) and (10), $\eta$ is the study speed.

In the errors reverse transmission, suppose that $g_{k}$ is the minus grade change direction when it is iterated for the $k$ th time, $p_{k}$ is the conjugate grade change direction when it is iterated for the $k$ th time.

At the initial time, $p_{0}=-g_{0}$

When iterated every time, firstly searching $g_{k}$ along the minus grade direction, then searching along the conjugate grade direction. The conjugate grade direction is the linearity combination of the current conjugate grade direction and the former once conjugate grade direction.

$$
\begin{aligned}
& p_{k}=-g_{k}+\beta_{k} p_{k-1} \\
& p_{k}=-g_{k}+\beta_{k} p_{k-1}
\end{aligned}
$$

Among them:

$$
\begin{gathered}
\beta_{k}=\frac{\Delta g_{k-1}^{T} g_{k}}{g_{k-1}^{T} g_{k-1}} \\
\Delta g_{k-1}^{T}=\left(g_{k}-g_{k-1}\right)^{T}
\end{gathered}
$$

Self-fit study efficiency $\eta_{\mathrm{k}}$ is decided by the current network parameter, $\mathrm{S}$ is the network error curving surface.

$$
\begin{gathered}
\eta_{k}=\frac{E}{S} \\
E=\frac{1}{Q} \sum_{i=1}^{Q} E_{i}=\frac{1}{2 Q} \sum_{i=1}^{Q} \sum_{k=1}^{m_{3}}\left(y_{k}^{i}-a_{k}^{i}\right)^{2} \\
S=\sum_{k i=1}^{m_{2}} \sum_{l=1}^{m_{l}}\left(\frac{\partial E}{\partial \omega_{l k}}\right)^{2}+\sum_{k=1}^{m_{2}}\left(\frac{\partial E}{\partial n}\right)^{2} \\
+\sum_{l=1}^{m_{3}} \sum_{k=1}^{m_{2}}\left(\frac{\partial E}{\partial \omega_{l k}}\right)^{2}+\sum_{l=1}^{m_{3}}\left(\frac{\partial E}{\partial i_{E}}\right)^{2}
\end{gathered}
$$

\section{B. Execution Steps}

- The first step: Initialization. The random numbers are endowed to the threshold and weight modulus.

- The second step: Input the training samples, enactment the error precision, compute the output of each layer.

- The third step: Compute the network output error.

- The fourth step: Compute the error signals of each layer.

- The fifth step: Adjust each layer weight value.

- The sixth step: Estimate the samples average errors meet, if meet, study over.

- The seventh step: Estimate network training entering the extremum point either or not, if entering, the reverse transmit processed by the conjugate grads method, otherwise, the reverse transmit processed by the BP arithmetic.

- The eighth step: Estimate the samples average errors meet the error precision either or not, if meet, study over, otherwise, return to the third step.

\section{Missile Launcher Faults Prediction Example}

It is very complex and difficult to predict the electric devices faults [7]. If the blooey parts and reasons can be predict timely and diagnose accurately when the launcher isn't disassembled, the reliability and security of the devices will be improved greatly. The maintenance blindness and time is reduced. The missile launcher has a characteristic of nonlinear transfer, it is provided that the vibrancy work signals are unstabilized stochastic progress, and it is an effective prediction technique to predict faults by using the vibrancy work signals.

The vibrancy signals of each tested point can be gained by the piezoelectricity acceleration sensors, the vibrancy states are measured by the date manage system, the swing scope is adopted as the criterion which the devices are blooey or not. 6 test points are chose as the tested objects. The emulation training is implemented by the FNN toolbox of Matlab 6.5. The speed of FNN is obviously faster than the speed of BP. Commonly the speed of FNN is $1000 \sim 10000$ times of the BP, The training progress and the training speed of the two networks are shown in Fig.2.

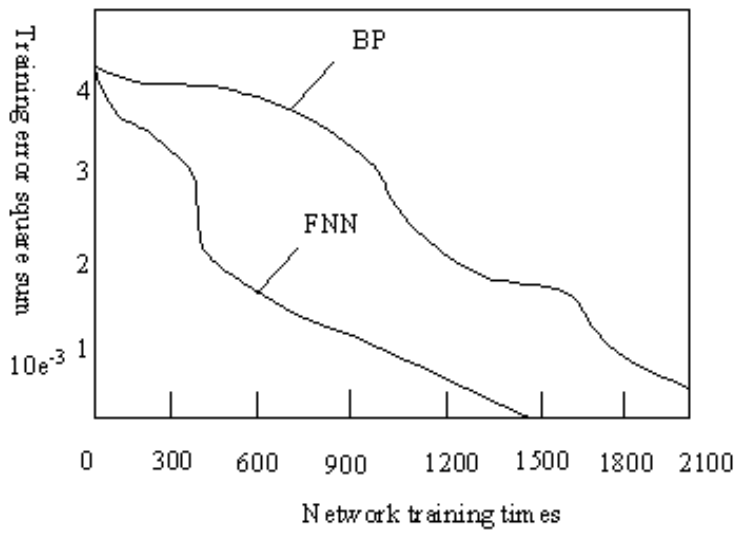

Figure 2. The speed curve of the two networks 
During the simulation experiments, 100 groups' simulation dates are obtained, the anterior 60 groups' dates are the training samples, the latter 40 groups dates are the test samples. The faults of the engine are identified by the trained model. The partial test results are shown in Table I.

TABLE I. THE PARTIAL TEST RESULTS

\begin{tabular}{|l|l|l|l|l|l|l|l|}
\hline & $\begin{array}{c}\text { Point } \\
\mathbf{1}\end{array}$ & $\begin{array}{c}\text { Point } \\
\mathbf{2}\end{array}$ & $\begin{array}{c}\text { Point } \\
\mathbf{3}\end{array}$ & $\begin{array}{c}\text { Point } \\
\mathbf{4}\end{array}$ & $\begin{array}{c}\text { Point } \\
\mathbf{5}\end{array}$ & $\begin{array}{c}\text { Point } \\
\mathbf{6}\end{array}$ & $\begin{array}{l}\text { Prediction } \\
\text { results }\end{array}$ \\
\hline 1 & 0.12 & 0.22 & 0.18 & 0.32 & 0.27 & 0.13 & Good \\
\hline 2 & 0.32 & 0.51 & 0.44 & 0.36 & 0.41 & 0.29 & $\begin{array}{l}\text { Low-degree } \\
\text { fault }\end{array}$ \\
\hline 3 & 0.36 & 0.68 & 0.55 & 0.49 & 0.52 & 0.71 & fault \\
\hline 4 & 0.89 & 0.72 & 0.62 & 0.92 & 0.88 & 0.58 & $\begin{array}{l}\text { high-degree } \\
\text { fault }\end{array}$ \\
\hline 5 & 0.94 & 0.66 & 0.85 & 0.76 & 0.77 & 0.64 & $\begin{array}{l}\text { high-degree } \\
\text { fault }\end{array}$ \\
\hline 6 & 0.25 & 0.13 & 0.15 & 0.21 & 0.30 & 0.08 & Good \\
\hline 7 & 0.37 & 0.42 & 0.28 & 0.42 & 0.38 & 0.26 & $\begin{array}{l}\text { Low-degree } \\
\text { fault }\end{array}$ \\
\hline 8 & 0.60 & 0.55 & 0.48 & 0.67 & 0.72 & 0.58 & fault \\
\hline 9 & 0.15 & 0.22 & 0.26 & 0.18 & 0.33 & 0.20 & Good \\
\hline 10 & 0.45 & 0.39 & 0.57 & 0.73 & 0.54 & 0.42 & fault \\
\hline
\end{tabular}

\section{CONCLUSIONS}

Based on the FNN technique, the faults prediction theory is introduced, the faults prediction analysis model is built, and an advanced training algorithm is proposed. Such algorithm is tested by using in the fault prediction of the missile launcher. The ability of the fault prediction is stronger. The faults prediction speed is faster $80 \%$ than ever; the fault prediction accuracy of this system is higher than ever. The cost of the system is cheaper $50 \%$ than ever. The experiment results illustrate that such method is better than traditional BP, and has good prospect.

\section{REFERENCES}

[1] J. Huang and J. F. Chen, "BP Neural Network model for early Diagnosis of Kawasaki Disease," Advanced Materials Research, vol. 2, no. 3, 2012, pp. 723-726.

[2] Zhang Chao, Xu Xjing and Shi Lijuan, "Fault Diagnosis System of Locomotive Alex's Track Based on Virtual Instrment," Proc. IEEE Computer Society. IEEE Press, July 2010, pp. 53-56.

[3] Chonggang Zhang and Minghui guo, "Application of the Fuzzy Theory in the expert system," China Mesurement and Test, vol. 34, no. 5,2008 , pp. $122-125$.

[4] Fang Zhiguang, Jiang Jianxun and Xu Jie, "Efficient Collision Detection Using a Dual K-DOP-Sphere Bounding Volume Hierarchy," Proc. IEEE Computer Society. IEEE Press, July 2010, pp. 185-189.

[5] Yufeng Zhu, Xiaoli Ding, Zhiwei Li and Shijian Zhou, "Analysis on Uranic Slope Stability Based on Neural Network," Proc. IEEE Computer Society. IEEE Press, July 2010, pp. 421-423.

[6] Chunyang Ma, Guo Li, "The Device Fault Prediction Research Based on The FNN," Yawp and Libration Control, vol. 6, no. 11, 2011, pp. 33-35.

[7] Peibin Chen, Zhiping Chen and Jianwen Qi, "Research of Airborn Radar Fanults Diagnosis Expert System Based on CBR," Measurement Technique, vol. 25, no. 1, 2008, pp. 9-11. 